\title{
Investigation of the Influence \\ of a Cavity Type Defect on the Frequencies of the Natural Rail Oscillations by the Radar Method
}

\author{
Vadim S. Potylitsyn, \\ Danil S. Kudinov and Ekaterina A. Kokhonkova* \\ Siberian Federal University \\ 79 Svobodny, Krasnoyarsk, 660041, Russia
}

Received 10.10.2019, received in revised form 04.12.2019, accepted 05.12.2019

Currently, the problem of non-destructive testing of rail lashes or rolling stock is quite acute, as the demands of both speed characteristics and vehicle safety increase every year. Thus, the purpose of this study was to determine the possibility of recording frequencies of natural oscillations by the radar method for rail lashes and the influence of the dimensions of the cavity-type defect. For this purpose, a laboratory bench was created with a standard rail, the length of $1.19 \mathrm{~m}$ of the P-65 brand, in which free oscillations were excited using a shock impulse load. Measurements of natural oscillation frequencies of the rail were recorded on a 24-bit ADC and a specialized accelerometer ZETLAB BC110. To register the oscillations, a Doppler radar with a frequency of $10 \mathrm{GHz}$ was used, which was installed at the focus of a parabolic mirror offset antenna with a 1.6-meter diameter. It was found that a "field" defect shifts the frequency mode of oscillation $750 \mathrm{~Hz}$ upwards. It is shown that the hole in the rail neck with a diameter of $10 \mathrm{~mm}$ makes a change in the frequency of about $14 \mathrm{~Hz}$.

Keywords: radar, flaw detector, rail, free oscillations, frequency shift.

Citation: Potylitsyn V.S., Kudinov D.S., Kokhonkova E.A. Investigation of the influence of a cavity type defect on the frequencies of the natural rail oscillations by the radar method, J. Sib. Fed. Univ. Eng. technol., 2019, 12(8), 1006-1012. DOI: 10.17516/1999-494X-0201.

(c) Siberian Federal University. All rights reserved

This work is licensed under a Creative Commons Attribution-NonCommercial 4.0 International License (CC BY-NC 4.0).

* Corresponding author E-mail address: markuss86@mail.ru 


\title{
Исследование влияния дефекта типа полости
}

\author{
на частоты свободных колебаний рельсов \\ радиолокационным методом
}

\author{
В.С. Потылицын, Д.С. Кудинов, Е.А. Кохонькова \\ Сибирский федеральный университет \\ Россия, 660041, Красноярск, пр. Свободньий, 79
}

\begin{abstract}
В настоящее время проблема неразрушающего контроля рельсовых плетей или подвижного состава стоит достаточно остро, так как с каждым годом возрастают требования как $\kappa$ скоростным характеристикам, так и к безопасности на транспорте. Таким образом, целью настоящего исследования являлось определение возможности регистрации частот собственных колебаний радиолокационным методом для рельсовых плетей и влияние размеров дефекта типа «полость». Для этого был создан лабораторный стенд с установленным стандартный рельсом длиной 1.19 м марки Р-65, в котором при помощи ударной импульсной нагрузки возбуждались свободные колебания. Измерения частот собственных колебаний рельса регистрировали на двадиатичетырехразрядный АЦП и специализированный акселерометр ZETLAB BC110. Для регистраџии колебаний использовали доплер радиолокатор частотой 10 ГГи, установленный в фокус параболической зеркальной офсетной антенны диаметром 1.6 м. Было выявлено, что дефект типа «полость» сдвигает частотную моду колебаний 750 Ги вверх. Показано, что отверстие в шейке рельса диаметром 10 мм вносит изменение частоть порядка 14 Ги.
\end{abstract}

Ключевые слова: радиолокатор, дефектоскоп, рельс, свободные колебания, частотный сдвиг.

В настоящее время существет много способов проведения дефектоскопии промышленных объектов, как, например, визуальные, ультразвуковые, радиолокационные, лазерные и др. Визуальные методы в основном используют для регистрации внешних дефектов, для внутренних дефектов применимы ультразвуковые и радилокационные [1-3]. Для дефектоскопии промышленных объектов, в том числе железнодорожного транспорта, в Российской Федерации наиболшее распространение получили методы, основанные на ультразвуковом исследовании, но данная методика имеет ограничения по скорости проведения диагностики и глубине проникновения ультразвуковых волн, что влечет за собой снижение производительности работ и невозможность определения дефекта типа «трещина» с той стороны объекта иследования, куда не проникают ультразвуковые волны $[4,5]$. Визуальные методы также имеют практически те же недостатки, что и ультрозвуковые, но при этом не позволяют обнаруживать внутренние деффекты типа «полость» [6]. Таким образом, радиолокационный метод исключает эти недостатки, так как любой дефект приводит к смещению частоты собственных колебаний рельса, при этом разные типы дефектов оказывают различное влияние на смещение данных частот, а возникновение нового дефекта вызывает образование новых колебательных мод из-за стоячих волн.

В данной статье приведены экспериментальные данные, полученные с использованием радиолокационного метода для дефекта типа «полость». Этот метод основан на эффекте изменения частоты собственных колебаний рельса в зависимости от наличия и размера дефекта. На рис. 1 показан сдвиг частоты собственных колебаний рельса в зависимости от наличия или 


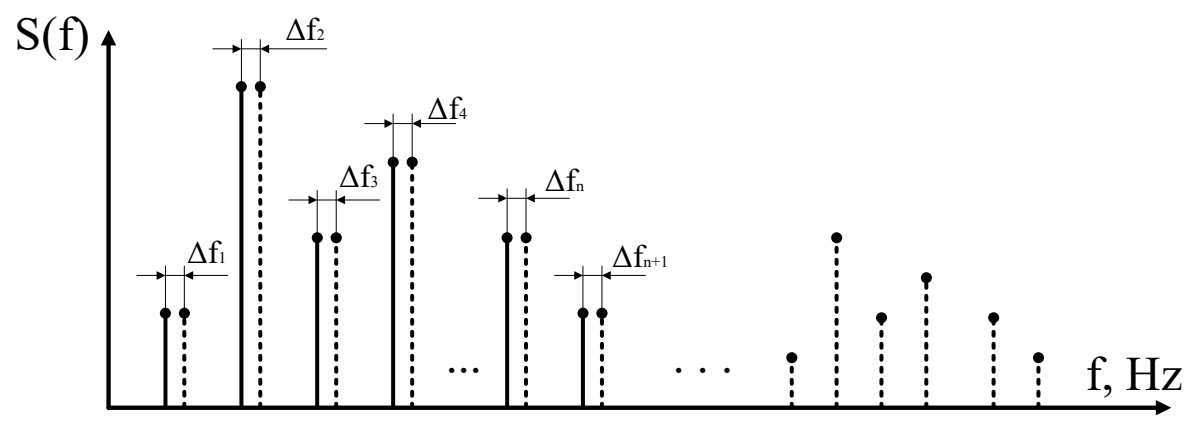

Рис. 1. Принцип выявления дефекта путем регистрации дополнительных колебательных мод в спектре собственных колебаний рельсов

Fig. 1. The principle of detecting a defect by registering additional vibrational modes in the spectrum of natural oscillations of rails

отсутствия дефекта типа «полость». Размеры дефекта тоже в свою очередь влияют на частотный сдвиг $\Delta \mathrm{f}$, а также добавляют частотные гармоники, обусловленные образованием дополнительных стоячих волн. В наших ранних работах мы уже публиковали данные при дефекте типа «трещина» [7].

\section{Методы и материалы}

Для физического моделирования использовали стандартный рельс длиной 1.19 м марки Р-65, в качестве подлегающего грунта был взят бак с песком размером 2.1х1.32х3.2 м. На рис. 2 приведена схема измерения. В данном случае измерения проводили как на двух опорах, расположенных на расстоянии 1.03 м, так и с имитацией междушпального пролета 0.46 м. Размеры деревянных брусков с квадартным сечением, имитирующих шпалы, составляли $32 \times 32 \mathrm{~cm}$.

Частоты собственных колебаний для размеров данного рельса равны для различных осей $\mathrm{f}_{\mathrm{x}}=750$ Гц и $\mathrm{f}_{\mathrm{y}}=1530$ Гц. В данном случае частоты были рассчитаны для стержня этих размеров, у рельса более сложная структура, и, следовательно, кроме этих колебательных мод он имеет дополнительные частотные колебательные моды.

Измерения параллельно проводили, как с использованием радиолокатора частотой 10 Ггц [8], так и при помощи акселерометра марки ZETLAB BC110 с осевой чувствительностью $104.8 \mathrm{mB} / \mathrm{g}$ и частотным диапазоном 0.5-10000 Гц. Радиолокатор находился в фокусе зеркала размером 1.6 м. Парабалическая оффсетная зеркальная антенна закреплялась на высоте 1.6 м. Мощность локатора, находящегося в центре зеркала, составляла 20 мВт.

Для того чтобы оценить мощность сигнала на входе локатора, воспользуемся стандартной формулой радиолокации, хорошо описанной в литературе:

$$
P_{R}=\frac{P_{T} \cdot G_{p} \cdot S_{A} \cdot e^{-2 \cdot \alpha \cdot r} \cdot \sigma}{4 \cdot \pi \cdot r^{4} \cdot \lambda^{2}},
$$

где $P_{R}$ - мощность сигнала на входе приемника; $r$ - растояние от антенны до объекта исследования; $\alpha$ - коэффициент поглощения радиоволн средой, в которой распространяется сигнал; $P_{T}-$

$$
-1008-
$$




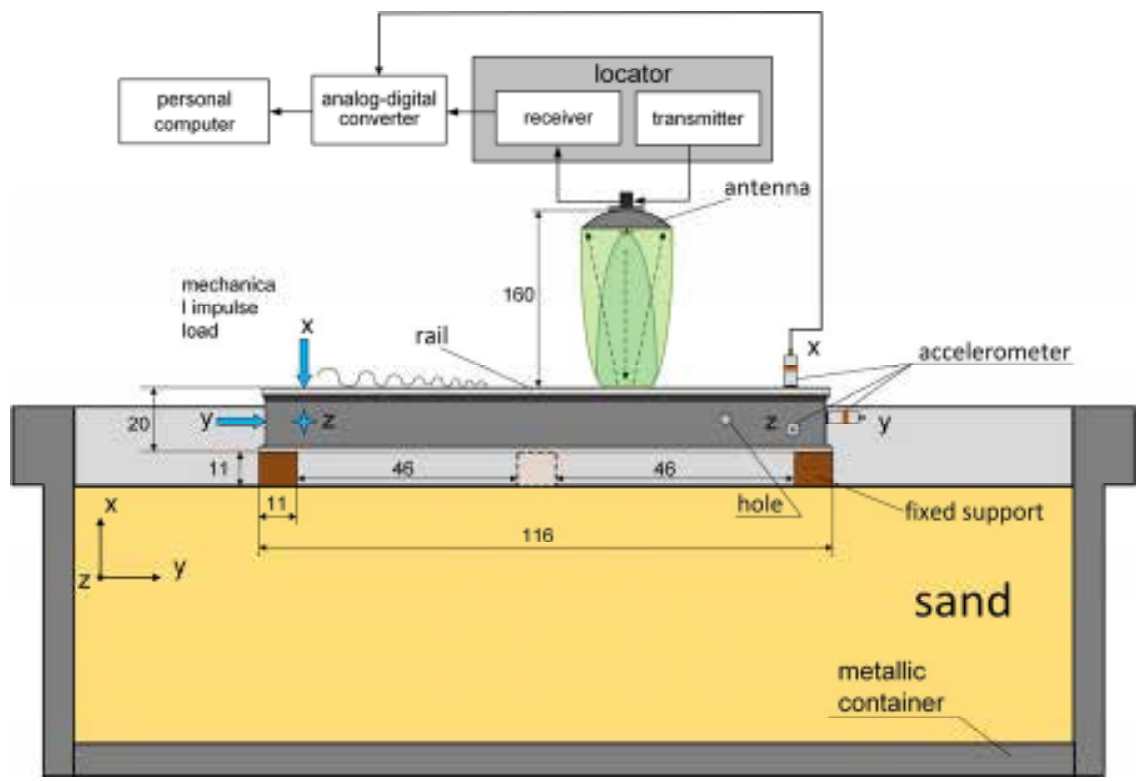

Рис. 2. Схема проведения эксперимента

Fig. 2. Scheme of the experiment

мощность передатчика; $G_{p}=0.65 \cdot \frac{4 \cdot \pi \cdot S_{A}}{\lambda^{2}}-$ коэффициент направленности антенны; $\lambda$ - длина волны; $\sigma=\frac{4 \cdot \pi \cdot S^{2}}{\lambda^{2}}-$ эффективная площадь рассеяния рельса; $S_{A}-$ эффективная площадь апертуры передающей или приемной антенны.

Диаметр пятна, которое создает антенна на расстоянии 1.6 м, таков:

$$
d=2 \cdot r \cdot \operatorname{tg}\left(\frac{70 \cdot \lambda}{2 \cdot L}\right)=2 \cdot 1.6 \cdot \operatorname{tg}\left(\frac{70 \cdot 3 \cdot 10^{-2}}{2 \cdot 1.6}\right) \approx 0.034 \mathrm{м} .
$$

Таким образом, будет обеспечиваться полное отражение от головки рельса и мощность на выходе приемной антенны будет составлять

$$
P_{R}=\frac{P_{T} \cdot G_{p} \cdot S_{A} \cdot e^{-2 \cdot \alpha \cdot r} \cdot \sigma}{4 \cdot \pi \cdot r^{4} \cdot \lambda^{2}} \approx 1.2 \cdot 10^{-6} \mathrm{Bm}
$$

Для регистрации колебания рельса использовали доплер локатор, и напряжение на его выходе составляло $\approx 1.16 \mathrm{мB}$, что при современной элементной базе позволило сделать низкочастотный усилительный тракт с коэффциентом усиления $\mathrm{K}_{\mathrm{u}}=5000$; далее сигнал подавали на (24-разрядный) ADC производства фирмы LCard - LTR-CEU-1-4 с установленным модулем LTR24. Частота дискретизации составляла 19 кГц, напряжение опоры $\pm 10 \mathrm{~B}$; учитывая это, шаг квантования составлял 1.2 мкВ, что в данном случае позволяло регистрировать колебания рельса с достаточным разрешением по напряжению. Запись сигналов проводили при помощи пакета программ LGraph, а дальнейшая обработка, в том числе и вычисление преобразования Фурье, при помощи Matlab. 
Для моделирования дефекта типа «полость» использовали отверстие, просверленное в шейке рельса с различными диаметрами: 1.5, 3, 4.8, 6 и 10 мм. Данное отверстие находилось на расстоянии 9 см от правого края и 4 см от верхнего края головки рельса. Рельс возбуждался ударной импульсной нагрузкой в различных направлениях как по осям х, y, z, так и по всем осям сразу.

\section{Результаты и обсуждение}

На рис. 3 изображены спектры сигналов, полученные при помощи радиолокатора и акселерометра, закрепленного сверху на головке рельса. На рис. $3 a, b$ приведены данные, полученные на рельсе без отверстия; в этом случае частота колебательной моды составила $\approx 747.5$ Гц, при наличии отверстия диметром 10 мм частота данной моды равнялась $\mathrm{f} \approx 761.5$ Гц, т.е. на данной моде колебаний отверстие диаметром 10 мм дает частотный сдвиг $\Delta \mathrm{f} \approx 14$ Гц.

В этом случае приведены данные только в вертикальной оси х, регистрация же колебательных мод, соответствующих другим осям (z, y), происходила, но уже с меньшими амплитудами. Установка акселерометра в разных плоскостях позволила регистрировать дополнительные частотные моды, которые не отмечал радиолокатор. Был зарегистрирован частотный сдвиг только одной колебательной моды, соответствующей колебаниям рельса в плоскости Ү. С точки зрения теории изменение частоты колебательных мод и появление новых за счет образования дополнительных стоячих волн должны происходить в плоскостях, перпендикулярных данному отверстию, т.е. в плоскостях Ү и Х. Сдвиг колебательной моды в плоскости Х не был зарегистрирован локатором и акселерометром, который был установлен в разных плоскостях.

По мнению авторов, данный эффект может быть обусловлен аномалией, связанной конкретно с этим экземпляром рельса. Это требует дополнительных исследований данного эффекта. На частотах выше 7 кГц возникают дополнительные моды, обусловленные наличием дефекта, его положением относительно краев рельса. В данной статье мы лишь указываем на их появление. В дальнейших работах мы рассмотрим этот вопрос более детально. На рис. 4 приведена зависимость сдвига частотной колебательной моды $\mathrm{f}_{\mathrm{x}} \approx 750$ от размера моделирующего дефекта.

Как видим, на графике рис. 4 наблюдается практически прямая зависимость частотного сдвига от размеров моделирующего дефекта за исключением небольших отклонений от этой величины. В более ранних работах мы приводили данные, что при дефекте типа «трещина» частоты колебательных мод уменьшаются. Учитывая все вышесказанное, имеется возможность не только производить обнаружение дефекта типа «полость», но и определять его размеры и положение относительно краев рельса за счет определения частот колебательных мод, обусловленных образованием дополнительных стоячих волн.

\section{Заключение}

Таким образом, можно сделать вывод, что для регистрации дефектов типа «полость» наиболее подходящим является диапазон частот около 10 ГГц, который позволяет регистрировать колебания рельса с требуемой точностью, без существенного влияния погодных условий. Было показано, что дефекты такого типа могут регистрироваться при помощи радиолокатора за счет сдвига частотной моды колебаний вверх. При дефектах типа «трещина» происходит сдвиг не- 

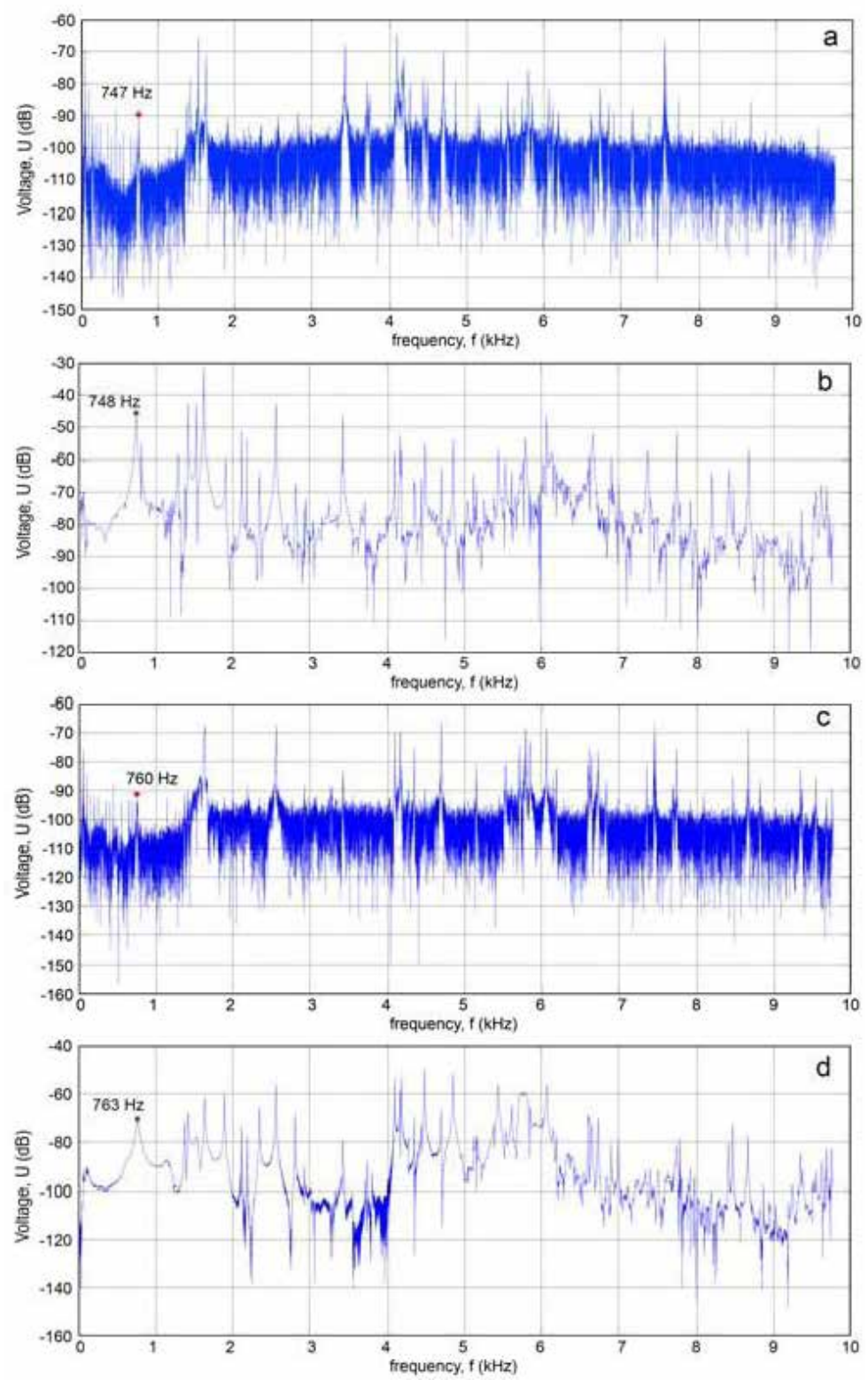

Рис. 3. Спектры сигналов: a - радиолокатор, рельс без отверстия; $\mathrm{b}$ - акселерометр, рельс без отверстия; c-радиолокатор, отверстие диметром 10 мм; d-акселерометр, рельс с отверстием диаметром 10 мм

Fig. 3. Signal spectra: a - radar, a rail without a hole; $b$ - accelerometer, a rail without a hole; $c-a$ radar, a hole with a diameter of $10 \mathrm{~mm} ; \mathrm{d}$ - an accelerometer, a hole with a diameter of $10 \mathrm{~mm}$

скольких частотных мод вниз и имеется возможность не только обнаружения, но и идентификации различных дефектов в рельсах. Данное утверждение требует дополнительного исследования с одновременным физическим моделированием дефектов типа «полость» и «трещина».

\section{Благодарности}

Эта работа выполнена при поддержке Российского фонда фундаментальных исследований (РФФИ), проект № 16-07-00426. 


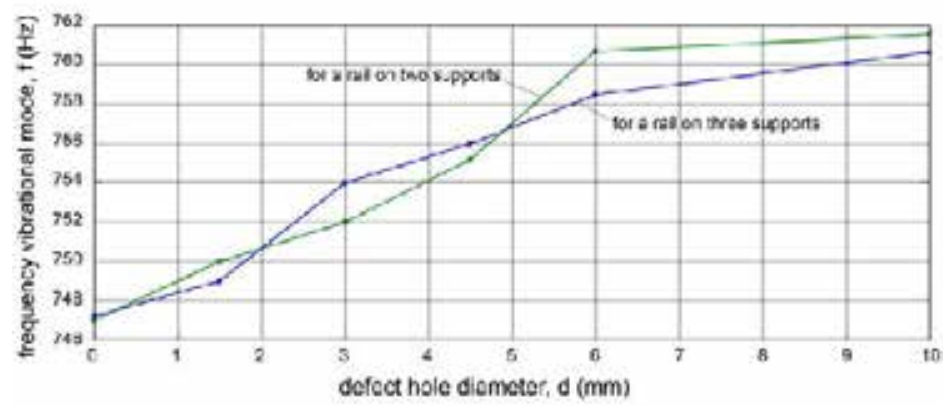

Рис. 4. Зависимость частоты колебательной моды от размера моделирующего дефекта

Fig. 4. Dependence of the frequency of the vibrational mode on the size of the modeling defect

\section{Список литературы}

[1] Moustakidis S., Kappatos V., Karlsson P., Selcuk C., Gan T. H., Hrissagis K. An Intelligent Methodology for Railways Monitoring Using Ultrasonic Guided Waves, Journal of Nondestructive Evaluation, 2014, 33(4), 694-710

[2] Zhao Y., Sun J. H., Ma J., Liu S., Guo R., Song J. F., Jia Z. Q. Application of the hybrid laser ultrasonic method in rail inspection, Insight - Non-Destructive Testing and Condition Monitoring, 2014, 56(7), 360-366

[3] Alemi A., Corman F., Lodewijks G. Condition monitoring approaches for the detection of railway wheel defects, Proceedings of the Institution of Mechanical Engineers, Part F: Journal of Rail and Rapid Transit, 2017, 231(8), 961-981

[4] Zhu L., Sun J., Dong M., Li G., Chen J., Niu S. Research of ultrasonic testing applied in rail flaw high-speed, Proceedings of the International Symposium on Precision Mechanical Measurement, 2002, 1, 176-180

[5] Krause H. J., Panaitov G. I., Zhang Y. Conductivity tomography for non-destructive evaluation using pulsed eddy current with HTS SQUID magnetometer, Applied Superconductivity Conference, 2002, 13(2), 215-218

[6] Liu Z., Li W., Xue F., Xiafang J., Bu B., Yi Z. Electromagnetic Tomography Rail Defect Inspection, IEEE Transactions on Magnetics, 2015, 51(10), 1-7

[7] Shaydurov G. Y., Kudinov D. S., Kokhonkova E. A., Potylitsyn V. S., Radar analysis of free oscillations of rail for diagnostics defects, Journal of Physics: Conference Series, 2018 1015(3), 032182

[8] Kudinov D. S., Potylitsyn V. S., Artemev K. A., Estimation of energy ratios and selection of optimal wavelength for radar flaw detector, Journal of Physics: Conference Series, 2018, 1015(3), 032183 